\title{
Faktor Mensch Teil 1 - vor-Corona-Phase
}

\author{
Stefan Reinheimer $\cdot$ Kristin Weber
}

(C) Springer Fachmedien Wiesbaden GmbH, ein Teil von Springer Nature 2020

Die etwas inhumane Betrachtung des Menschen als der Bereitsteller des Produktionsfaktors Arbeit, wie es die frühen Ökonomen Adam Smith und David Ricardo propagierten, ist nicht Gegenstand der vorliegenden Ausgabe der HMD. Vielmehr geht es darum, den Menschen in einem immer technischeren Produktions- und Lebensumfeld zu verorten - seinen Einfluss auf die IT, den Einfluss der IT auf den Menschen und die Herausforderungen, die die zunehmende Beschleunigung des Wandels mit sich bringt. Der Mensch kann in seinem IT-Umfeld viele Rollen einnehmen: er nutzt die IT zur Effizienzsteigerung und erweitert seine eigene Effektivität; er muss sich der Veränderung seiner eigenen Rolle durch die IT beugen und sich anpassen - dies erfolgt zunehmend schneller und erfordert hohe Flexibilität, Anpassungsbereitschaft und -fähigkeit; er missbraucht die IT zu seinem persönlichen Vorteil. All diese Aspekte sind Betrachtungsgegenstand der Beiträge des vorliegenden Schwerpunktheftes.

Die HMD 333 ist unserer Überzeugung nach nicht nur für Numerologen und Esoteriker interessant. Spricht der Laie gerne von einer Schnapszahl, so ist die 333 für Esoteriker eine Engelszahl, eröffnet also einen Kommunikationsweg zu den Schutzengeln - auch wenn das aus wissenschaftlicher Sicht sicherlich kritisch zu bewerten ist, freuen wir uns, wenn wir entsprechend ausgerichteten Lesern auch hiermit etwas Positives vermitteln können.

Lehrende Historiker eselbrücken - erlauben Sie uns diese Verbisierung eines Substantives - mit „333 - bei Issos Keilerei“ Wissen über die entsprechende Schlacht, in der Alexander der Große die Makedonier zu einem großen Sieg über Dareios III

\footnotetext{
S. Reinheimer $(\bowtie)$

BIK GmbH, Nürnberg, Deutschland

E-Mail: sr@bik.biz

K. Weber

Hochschule für angewandte Wissenschaften Würzburg-Schweinfurt, Würzburg, Deutschland

E-Mail: Kristin.Weber@fhws.de
} 
und sein persisches Heer geführt hat. Geschichtlich weniger vorbelastete Zeitgenossen müssen sich jetzt nur noch merken, dass es sich um das Jahr 333 VOR Christi Geburt handelt.

Für uns als Herausgeber hat die HMD 333 aber auch noch eine andere, sehr außergewöhnliche Bedeutung: es ist die Ausgabe, deren Endphase vor dem Redaktionsschluss in den nahezu weltweiten Shutdown durch die Corona-Krise (wissenschaftlich korrekt: COVID-19-Krise) fällt. Der Heftschwerpunkt „Faktor Mensch“ erlangt auch für die IT in dieser Zeit eine besondere Bedeutung und einen Wandel, der vor wenigen Monaten noch nicht absehbar war. Unsere Zielsetzung mit dem Themenschwerpunkt orientierte sich bei ihrer Konzeption daran, welche Rolle der Mensch in der sich extrem schnell verändernden IT-Umgebung einnimmt, bzw. einnehmen muss, mit welchen Ängsten und Herausforderungen er umgehen muss und wie man Risiken reduzieren bzw. ihnen begegnen kann. Die Beiträge, auf die wir gleich noch näher eingehen werden, nehmen daher im Wesentlichen auch diese soziotechnische Perspektive ein. Durch den kleinen aber höchst effektvollen (biologischen) Virus SARS CoV-2 dreht sich auf einmal die Perspektive um: die IT wird zu einem Retter, um nicht zu sagen, Heilsbringer, der synchrone Kommunikation weiterhin ermöglicht, ohne die biologischen Risiken klassischer Meetings in Kauf zu nehmen. Schnell kommen Thesen auf, dass die Krise einen signifikanten Schub für die Digitalisierung leistet und die Erfahrungen der letzten Monate eine nachhaltige Bedeutung für die Mensch-Maschine-Beziehung haben werden. Wir werden das in der IT-Community sicherlich beobachten und haben uns als Herausgebergremium darauf verständigt, die These zu überprüfen, indem wir diesem Thema ein Schwerpunktheft in der zweiten Hälfte des kommenden Jahres widmen werden - vielleicht lautet dann der Titel des Editorial „Faktor Mensch Teil 2 - post-Corona-Phase“. Bis dahin sollten Forschung, Lehre und Praxis Implikationen abgeleitet, Konzepte entwickelt und Maßnahmen eingeleitet oder umgesetzt haben, so dass wir eine inhaltsschwere HMD zur IT in Krisenzeiten erwarten können - einen Titel dafür haben wir noch nicht fixiert.

Kommen wir nun aber zur aktuellen Ausgabe - „Faktor Mensch“ im IT-Verständnis der Vor-Corona-Zeit. Uns haben sehr viele Abstracts und Artikelvorschläge erreicht - weit mehr als üblich, so dass die Ausgabe 333 vom Umfang her den Durchschnitt übertrifft. Die Beiträge kommen aus Deutschland, Österreich und der Schweiz. Alle drei Fakten - Quantität, Qualität und geografische Vielfalt - freuen uns sehr. Jeder Beitrag greift dabei höchst praxisrelevante Aufgabenstellungen auf, manche über statistische Auswertungen, manche über Konzepte oder Projektbeispiele und manche regen über etwas philosophischere Ansätze zum Nachdenken an.

Unser Heft ist sehr informatikerfreundlich strukturiert - insgesamt $2^{4}$ Schwerpunktbeiträge lassen sich nach dem Überblicksbeitrag grob in $2^{2}$ Cluster gruppieren. Den Anfang macht wie immer ein einleitender Überblicksbeitrag, der unser Schwerpunktthema aus einer größeren Flughöhe beleuchtet und einstimmt und somit keiner Themengruppe zuzuordnen ist.

Die von der Anzahl der Beiträge umfangreichste Gruppe mit insgesamt sechs Artikeln könnte mit dem Schlagwort Praxisbeispiele und Implikationen umschrieben werden. Konzepte und Technologien werden auf ihre Auswirkungen auf den Men- 
schen untersucht. Dabei geht es im Wesentlichen um die Potenziale, die mit den diskutierten Ansätzen erschlossen werden können. Das Spektrum erstreckt sich von einem zeitgemäßen Interaktionsansatz für digitale Services und Motivationsansätze für die Digitalisierung in der Verwaltung über Technologien wie Chatbots, Virtual und Augmented Reality bis hin zu einer statistischen Auswertung des Arbeitsmarktpotenzials für die Digitalisierungsanforderungen im Umfeld der Künstlichen Intelligenz.

Unser nächster Aufsatz steht ein wenig allein und beleuchtet unter dem Stichwort Ethik die hier fast wörtlich zu nehmenden Einflüsse von Technik im Körper und ihre Möglichkeiten - Cyborg 1.0?

Das Zeitalter der Digitalisierung erfordert geistige Flexibilität und die Bereitschaft umzudenken und zu lernen. Das dritte Cluster - Training - widmet sich den schulungs- und weiterbildungsbezogenen Aufgaben, um den Menschen in seine zunehmend technologisierte Umwelt einzubetten und ihn „mitzunehmen“, wie es immer wieder anschaulich gefordert wird.

Fünf Beiträge unter dem Schlagwort Informationssicherheit bilden den abschlieBenden Block des Heftes „Faktor Mensch“. Datensouveränität, Angriffe auf die Sicherheit sozio-technischer Systeme, Cyber Security, Spear Phishing und die Rolle der eigenen Mitarbeiter im unternehmerischen Sicherheitskonzept sind die grundlegenden Schlagworte der Artikel in diesem Cluster, das die Risiken eines zunehmend datengetriebenen und datenbestimmten Lebens und Arbeitens thematisiert.

Zusätzlich zu unseren Schwerpunktbeiträgen haben wir in der Rubrik „Rezensionen“ zwei Buchbesprechungen im thematischen Umfeld unseres Heftes. Wir freuen uns, wenn Ihnen auch diese Beiträge einen Mehrwert bringen, indem sie Ihre Entscheidungsfindung unterstützen, diese Bücher eventuell in Praxis, Forschung oder Lehre nutzbringend einzusetzen.

Wir haben Ihnen einen umfangreichen Band mit einer thematischen breit gefächerten Vielzahl von Beiträgen zum Thema „Faktor Mensch“ zusammengestellt. Wir bedanken uns bei allen Autoren, die ihr Wissen und ihre Erkenntnisse mit uns allen teilen und würden uns freuen, wenn Sie viele Anregungen für Ihren Arbeitsalltag erhalten. Mit den der Zeit angepassten Grüßen „Bleiben Sie gesund“ wünschen wir Ihnen jetzt viel Spaß beim Lesen.

Stefan Reinheimer

Kristin Weber 